Article

\title{
Palladium Nanoparticles Tethered in Amine-Functionalized Hypercrosslinked Organic Tubes as an Efficient Catalyst for Suzuki Coupling in Water
}

\author{
Arindam Modak ${ }^{1,2}$, Jing Sun ${ }^{1}$, Wenjun Qiu ${ }^{1}$ and Xiao Liu ${ }^{1, *}$ \\ 1 Key Laboratory for Green Chemical Technology of Ministry of Education, School of Chemical Engineering \\ and Technology, Tianjin University, Tianjin 300072, China; arindam_modak_2006@yahoo.co.in (A.M.); \\ dugufanying@163.com (J.S.); wingqiu85@163.com (W.Q.) \\ 2 Indian Association for the Cultivation of Science, Jadavpur, Kolkata-700032, India \\ * Correspondence: liuxiao71@tju.edu.cn; Tel./Fax: +86-22-2789-0859
}

Academic Editor: Ioannis D. Kostas

Received: 7 September 2016; Accepted: 14 October 2016; Published: 20 October 2016

\begin{abstract}
It is highly desirable to design functionalized supports in heterogeneous catalysis regarding the stabilization of active sites. Pd immobilization in porous polymers and henceforth its application is a rapidly growing field. In virtue of its' scalable synthesis and high stability in reaction conditions, amorphous polymers are considered an excellent scaffold for metal mediated catalysis, but the majority of them are found as either agglomerated particles or composed of rough spheres. Owing to several important applications of hollow organic tubes in diverse research areas, we aimed to utilize them as support for the immobilization of Pd nanoparticles. Pd immobilization in nanoporous polymer tubes shows high activity in Suzuki cross coupling reactions between aryl halides and sodium phenyl trihydroxyborate in water, which deserves environmental merit.
\end{abstract}

Keywords: porous organic tubes; heterogeneous catalysis; Suzuki coupling in water

\section{Introduction}

Porous organic polymers (POPs) are emerging as next-generation support materials for heterogeneous catalysis [1-6]. Cheap and readily available organic precursors, tailorable functionality arising from diverse building blocks, are generally advantageous for making high surface area POPs, which are not only used as "support" for metal-mediated catalysis, but also possess significant applications in diverse research, owing to the advantages of densely packed organic groups [7-9]. Therefore, it is customary to mention that the inherent advantages of having organic units in POPs is tremendous, as a support for metal complexes/nanoparticles or as a catalyst because of the virtue of having an electronic interaction between the organic units in POPs and metal nanoparticles. In fact, the main advantage of POPs with its competitive porous support viz. metal organic frameworks (MOFs) [10] and periodic mesoporous organosilica (PMOs) [11,12] is its stability in drastic reaction conditions, which has been immensely highlighted as POPs have shown usability in water medium for catalysis.

Considering adverse environmental impact, using volatile organic solvent for catalysis is a serious concern, which should be replaced by water as an environmentally more demanding. However, reactions using water as the only solvent is frequently encountered as fatal because of the moisture sensitive organic precursors, catalysts, and intermediates. Therefore, it is highly challenging to develop water-compatible catalysts that could be stable, active, and reusable for a number of times without being deteriorated [13,14]. In this context, a Suzuki-Miyaura cross coupling reaction in 
water as an eco-friendly solvent is meritorious because of its high stability and good solubility of phenyl boronic acid/salt [15]. Pd-catalyzed Suzuki-Miyaura cross coupling reactions have significant importance in organic chemistry, pharmaceuticals research, drug discovery, and development as an elegant tool for $\mathrm{C}-\mathrm{C}$ bond formation reactions, mainly because of the wide availability of starting materials and relatively mild reaction conditions $[16,17]$. Although a majority of research has been done in homogeneous conditions using either organic/water as solvent, it suffers from the formation of Pd black as an inactive catalytic species. Again, the use of triphenyl phosphine for stabilization of Pd intermediates under homogeneous conditions often encounters toxicity/poison [18]. In this regard, high surface area heterogeneous catalysts possess tremendous applications in fine chemical industries, owing to its repetitive usability, tailorability of surface modification for stabilization of active sites, and so on. Pd-grafted heterogeneous catalysts comprising porous silica, zeolite, MOFs, and polymers are hereby reported as solid-supported catalysts for Suzuki reaction, but few show considerable recyclability and stability in a water medium [19-21]. On the other hand, microporous POPs are generally formed through precipitation polymerization as agglomerated solid particles/irregular flakes, instead of any well defined nanostructure [22]. Nevertheless, POPs having uniform morphology of hollow tubes/fibers are still scarce; moreover, their application is merely limited to device manufacturing [23]. Recently, Modak and Bhaumik reported interesting microporous polymer tubes (PP-1, PP-2, PP-3; PPs) that are uniform and show high heterogeneity for one-pot tandem catalysis [24]. However, PPs could also be interesting as support for metal nanoparticles' owing to their amine functionality; therefore, we investigated its activity for Suzuki-Miyaura cross coupling reactions. The catalysis in water might be advantageous, since the hydrophobic 4-tritylaniline-based tubes are stable and prevent the active sites from agglomeration and inactivation. Hopefully, this research can provide tremendous scientific interest in the utilization of highly functionalized porous organic tubes/fibers as support of nanoparticles.

\section{Results and Discussion}

\subsection{Synthesis and Characterization of Pd/PP-3 Tubes}

The amine-functionalized hypercrosslinked polymer (PP-3) is formed through a one-pot polymerization condensation using 4-Tritylaniline as starting precursor, dimethoxymethane (DMM) as linker and $\mathrm{FeCl}_{3}$ as catalyst/mediator (Scheme 1).

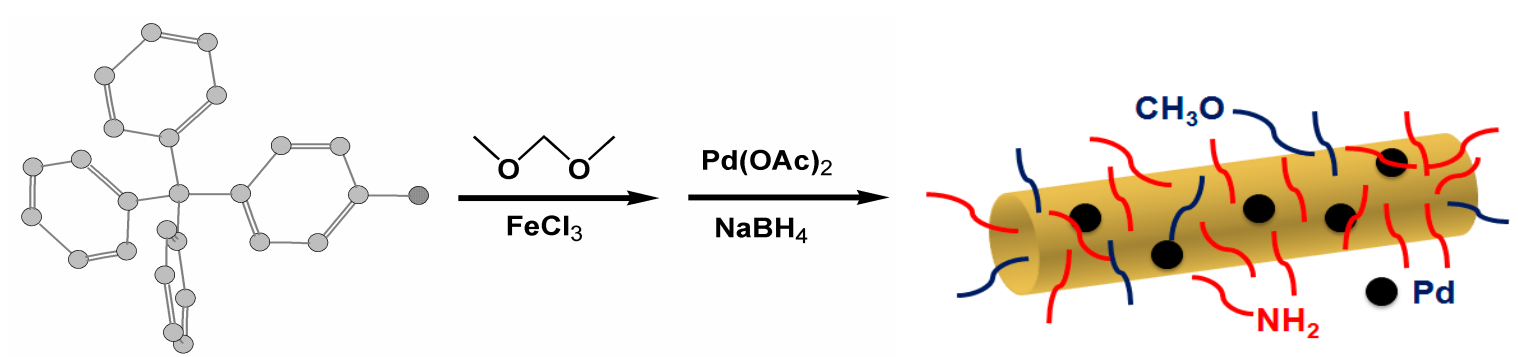

4-Tritylaniline

Scheme 1. Schematic representation for the formation of Pd/PP-3 from a 4-tritylaniline precursor.

The resulting light brown precipitate shows a unique hollow tube shaped morphology as shown in both scanning electron microscopy (SEM) and transmission electron microscopy (TEM) images (Figure 1). Unlike other tube-shaped porous polymers, synthesis of PP-3 is performed in relatively mild conditions utilizing $\mathrm{FeCl}_{3}$ as a cheap and non-harmful chemical $[23,25]$. The size of the organic tubes are found to be $\sim 5-7 \mu \mathrm{m}$ in length, and the inner hollow diameter is $\sim 80-100 \mathrm{~nm}$, together with $\sim 300-350 \mathrm{~nm}$ is the wall thickness. This thickness accounts for excessive polymerization/non-covalent interaction, which is due to the addition of a large quantity of DMM linker during the synthesis [24]. 
Pd immobilization to PP-3 was performed by loading with $\mathrm{Pd}(\mathrm{OAc})_{2}$, followed by a reduction with aqueous $\mathrm{NaBH}_{4}$ under mild conditions. It was observed in TEM images (Figure 2) that Pd nanoparticles with dimensions of 5-9 $\mathrm{nm}$ were distributed throughout PP-3, which is possibly because of the stabilization by built-in amine sites (Figure 2) [26-29].
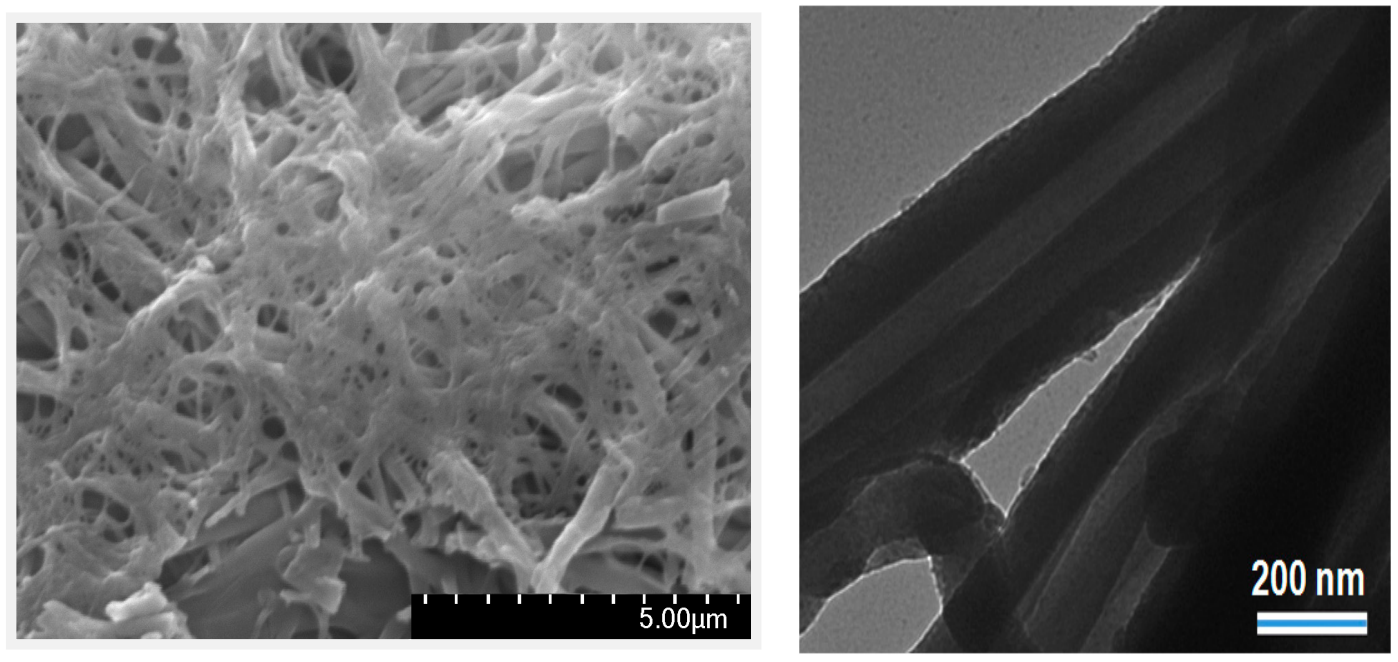

Figure 1. Scanning electron microscopy (SEM) (left) and transmission electron microscopy (TEM) (right) images of PP-3 tubes (Scale $200 \mathrm{~nm}$ for TEM).

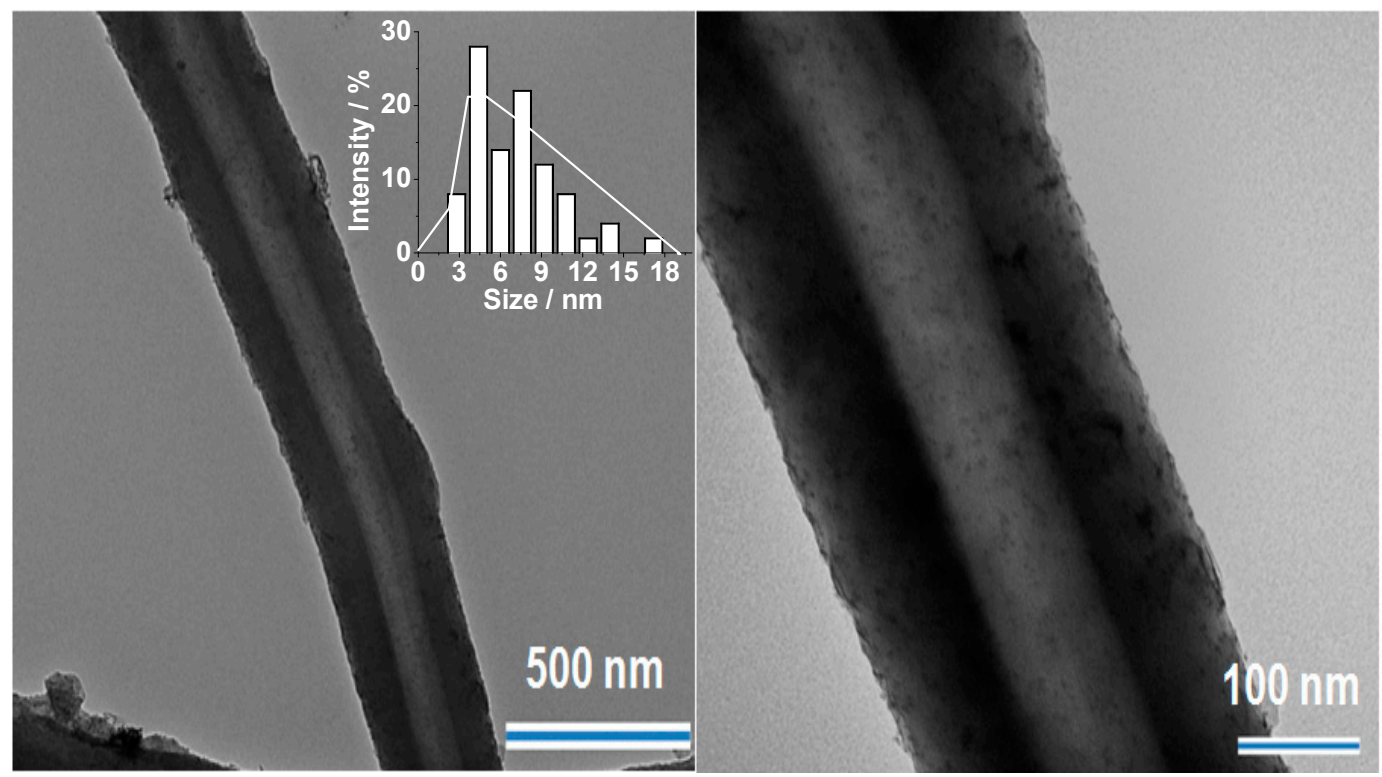

Figure 2. TEM images of Pd/PP-3.

Size distribution of Pd nanoparticles is shown in the inset of Figure 2, suggesting a broad distribution pattern (3-9 nm). Further characterization of Pd/PP-3 was achieved through powder X-ray diffraction (XRD), $\mathrm{N}_{2}$-sorption, $\mathrm{X}$-ray photoelectron spectroscopy (XPS), and inductively coupled plasma atomic emission spectroscopy (ICP) analysis and provides good justification for the presence of Pd nanoparticles in our hypercrosslinked PP-3 tubes.

PXRD of Pd/PP-3 is given in Figure 3a, which shows sharp diffraction at the $40.2^{\circ}, 43.9^{\circ}$, and $47.3^{\circ}$ regions, corresponding to different facets of Pd crystal particles. In comparison with Pd/PP-3, only PP-3 shows a broad peak because of the presence of an amorphous pore wall [30]. 
Porous properties of $\mathrm{Pd} / \mathrm{PP}-3$ were measured from the $\mathrm{N}_{2}$ sorption isotherm, as shown in Figure $3 b$, which preferentially suggests Type I characteristics of the isotherm. Like other microporous materials, a high uptake at a low $\mathrm{P} / \mathrm{P}_{0}$ is observed, followed by a flat extrapolation at $0.2-0.8 \mathrm{P} / \mathrm{P}_{0}$, along with a step uptake at $0.9 \mathrm{P} / \mathrm{P}_{0}$. The increase of the isotherm at $0.9 \mathrm{P} / \mathrm{P}_{0}$ depicts the inter-particle mesoporosity. The Brunauer-Emmett-Teller (BET) surface area of Pd/PP-3 was calculated to be $420 \mathrm{~m}^{2} \cdot \mathrm{g}^{-1}$, lower than $530 \mathrm{~m}^{2} \cdot \mathrm{g}^{-1}$ for PP-3 tubes, which is basically due to pore blocking by Pd nanoparticles. In Figure 4a, we provide a survey XPS spectrum of Pd/PP-3, which shows the presence of $\mathrm{N}, \mathrm{O}$, and $\mathrm{Pd}$. Figure $4 \mathrm{~b}$ shows the XPS of Pd $3 \mathrm{~d}$ electrons, which indicates that Pd sites were reduced to a metallic state in PP-3 with the presence of $\mathrm{NaBH}_{4}$. The confirmation of $\mathrm{Pd}(0)$ has been characterized at 334.4-334.8 eV and 339.8-340.2 eV, which were assigned to the $\mathrm{Pd} 3 \mathrm{~d}_{5 / 2}$ and $\mathrm{Pd} 3 \mathrm{~d}_{3 / 2}$ electrons, respectively [31,32]. Traces of a PdO peak at $341 \mathrm{eV}$ have been observed [33]. All these results, however, demonstrate almost a complete conversion of $\mathrm{Pd}(\mathrm{OAc})_{2}$ into $\mathrm{Pd}$ nanoparticles with the utilization of hollow PP-3 tubes.

(a)

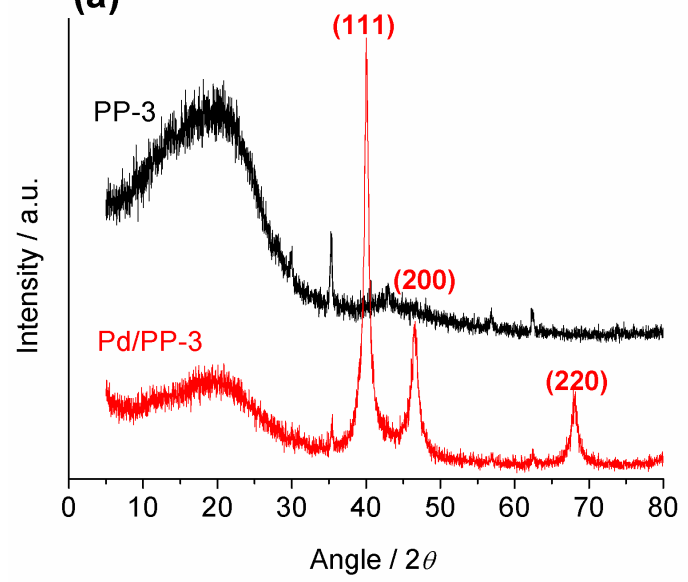

(b)

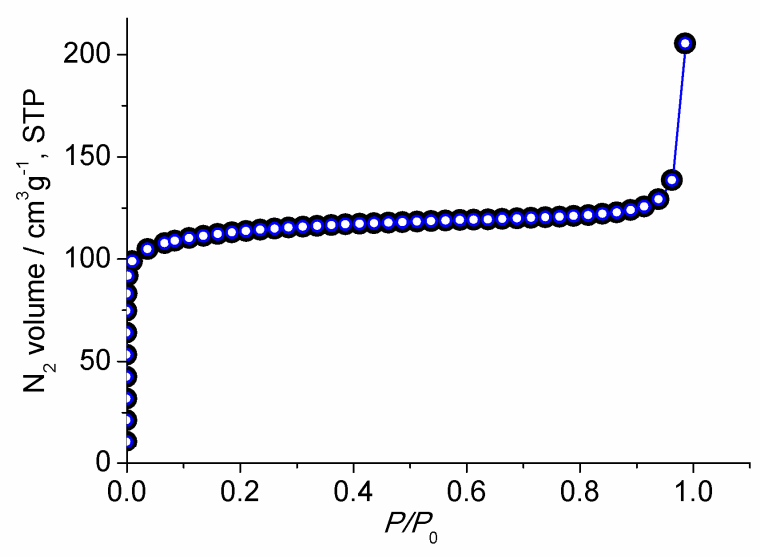

Figure 3. (a) Powder X-ray diffraction pattern; (b) $\mathrm{N}_{2}$ adsorption-desorption isotherm of Pd/PP-3.

(a)

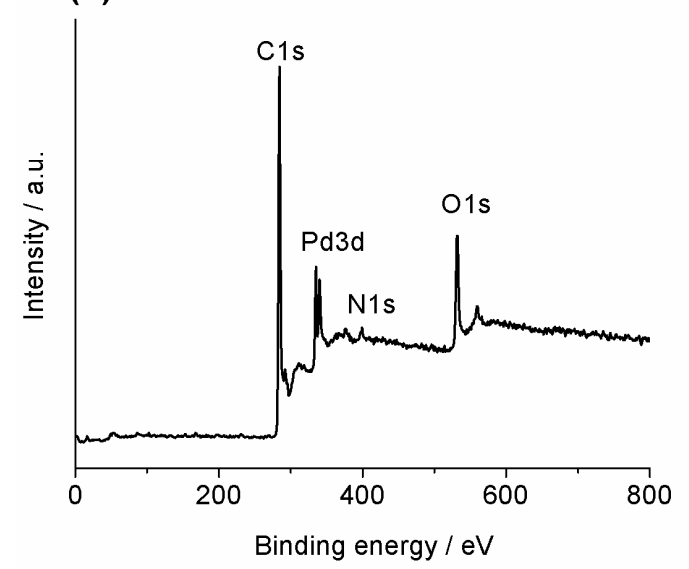

(b)

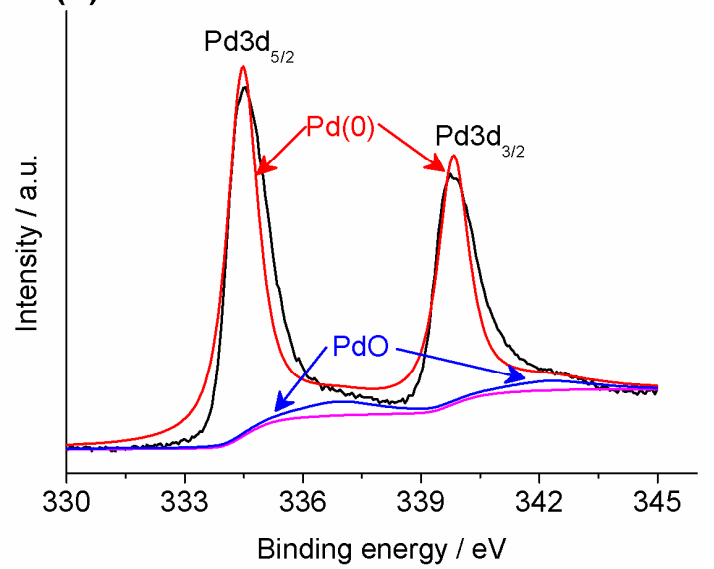

Figure 4. (a) Survey XPS (X-ray photoelectron spectroscopy) spectra of Pd/PP-3; (b) Deconvoluted XPS spectra of Pd 3d electrons in Pd/PP-3, showing the presence of Pd nanoparticles.

\subsection{Heterogeneous Catalysis for Suzuki-Miyaura Cross Coupling Reaction in Water}

Based on this perspective, the benchmark Suzuki reaction between bromobenzene and sodium phenyltrihydroxyborate was tested in water as a solvent at $100^{\circ} \mathrm{C}$. Because of the better solubility of sodium phenyltrihydroxyborate (PHB) in water compared with phenylboronic acid, the use of PHB for 
Suzuki coupling without the aid of an additional base was considered advantageous. Owing to the easy preparation and highly stable $\mathrm{PHB}$ as organoboron salt, $\mathrm{Pd}-$ mediated $\mathrm{C}-\mathrm{C}$ bond formation reactions are quite meritorious. [34]. Initially, we investigated the scope of Suzuki catalysis in several solvents such as toluene, dicholomethane, dioxane, water, and dimethylformamide (DMF) (Table 1). We observed that the reaction was very sluggish in non-polar, aprotic solvents such as toluene and dicholomethane, partly successful in 1,4-dioxane, and takes place quite efficiently in DMF and DMF/water mixtures (Table 1). Reaction in pure water shows only a $60 \%$ yield of biphenyl at $24 \mathrm{~h}$, which considerably improves to $>90 \%$ at $24 \mathrm{~h}$ upon the addition of tetrabutylammonium bromide (TBAB) as phase transfer catalyst.

Table 1. Optimization in reaction conditions for Suzuki coupling reactions between 1 equiv bromobenzene and 1.2 equiv sodium phenyltrihydorxyborate, catalyzed by Pd/PP-3 support.

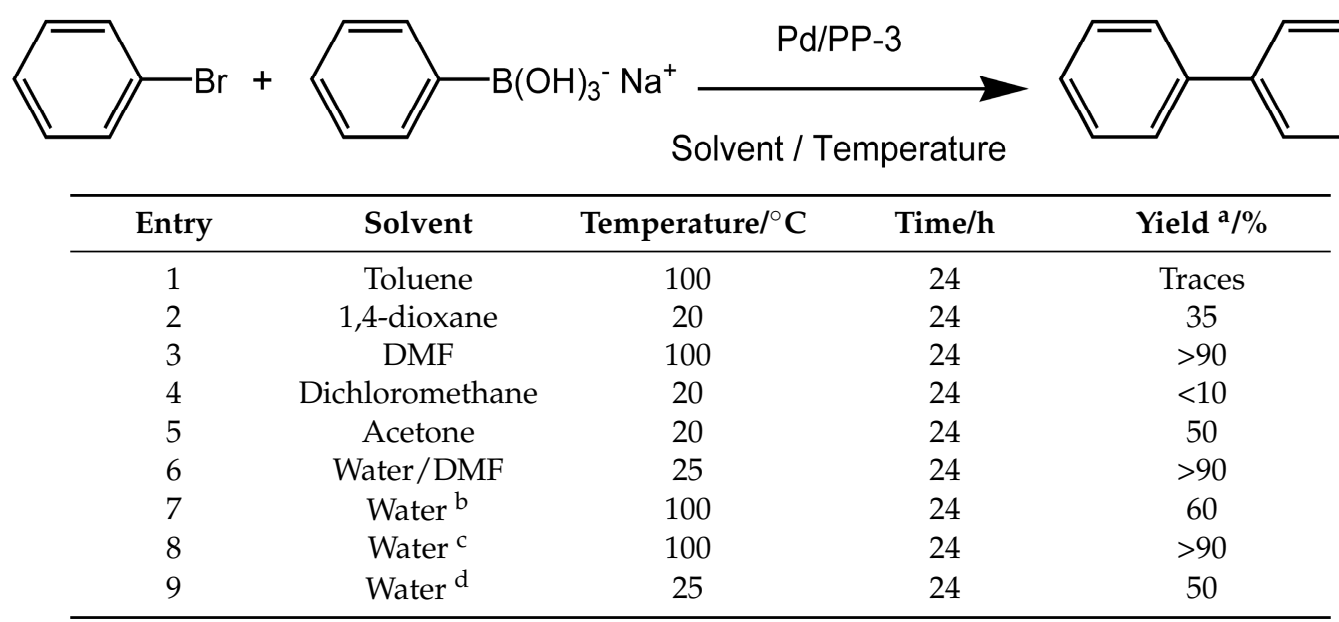

${ }^{a}$ Yield refers to isolated products after purification; ${ }^{\mathrm{b}}$ Without using TBAB; $^{\mathrm{c}, \mathrm{d}} 1$ equiv of TBAB.

Using TBAB during the reaction is essential in order to increase the solubility of organic precursors in water. However, upon the addition of $\mathrm{TBAB}$, the reaction can also take place at room temperature conditions, as shown in Table 1. In this regard, we show a temperature-dependent conversion of bromobenzene to biphenyl in Figure 5a, with or without TBAB. In the case of adding TBAB, our model reaction shows much faster kinetics than the reaction devoid of TBAB, demonstrating that phase transfer catalyst is indeed essential for reactions in water. Furthermore, the optimum loading of $\mathrm{Pd}(\mathrm{OAc})_{2}$ and consequently the generation of $\mathrm{Pd}$ nanoparticles in PP-3 influences catalytic activity, and we observe the highest catalytic activity/reaction rate $\left(12.5 \mathrm{~h}^{-1}\right)$ with $2 \mathrm{wt} \% \mathrm{Pd} / \mathrm{PP}-3$, which shows the highest yield of biphenyl from bromobenzene (Figure $5 b$ ).

It is worthy to mention that the reaction was carried out in aerial conditions without using degassed water/co-solvent, which could partially lead to the formation of agglomerated Pd/Pd black, as this phenomenon is quite common with several known palladacycles [35]. Therefore, the problem of using Pd/PP-3 in recycling studies was outperformed when choosing degassed water under $\mathrm{N}_{2}$ prior to adding substrates and catalysts, which worked out well in our study.

Next, the efficiency of Pd/PP-3 was tested by encountering a broad substrate scope ranging from electron rich to electron poor aromatic halides, as given in Table 2. Aromatic iodo and/or bromo compounds worked efficiently for the formation of substituted biphenyl; however, for chloro derivates, a partially sluggish reaction was observed. Nonetheless, there are many reports for heterogeneous catalysts such as Suzuki coupling reactions, which can work efficiently with expensive aromatic iodo/bromo derivatives, [36], but very few catalysts can activate aromatic chloro compounds, which are cheap and have more economically feasible applications [31]. 

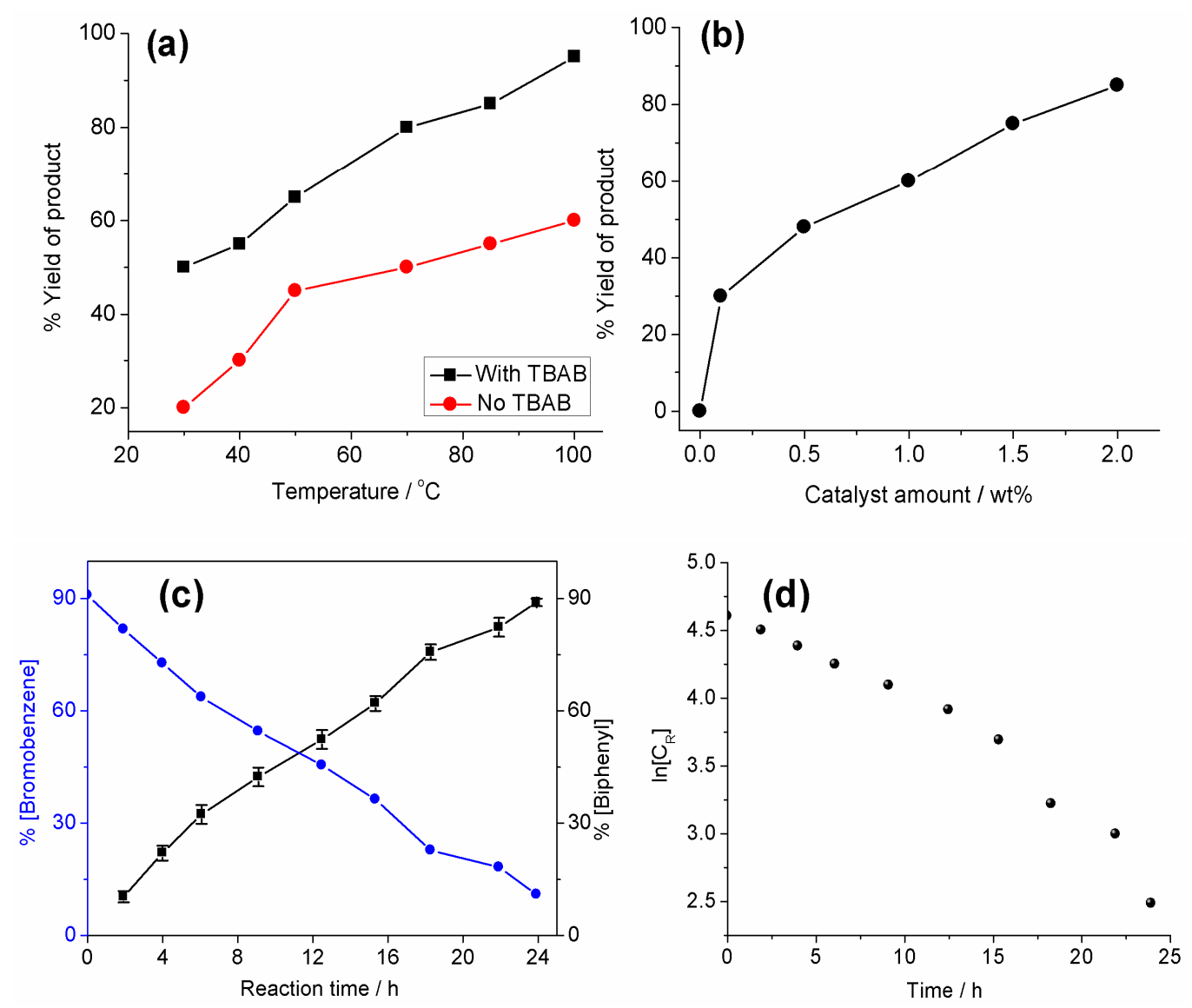

Figure 5. (a) Influence of reaction temperature; (b) Amount of catalyst on yield of biphenyl from bromobenzene; (c) Reaction profile/kinetic plot for bromoenzene conversion to biphenyl; (d) Plot of natural logarithm of the remained bromobenzene conc. during reaction with time.

Table 2. Substrate scope of Suzuki coupling reaction by $\mathrm{Pd} / \mathrm{PP}-3$. $^{\mathrm{a}}$

\begin{tabular}{cccccc} 
& & & & \\
\hline
\end{tabular}

a $1 \mathrm{mmol}$ aryl halide, $1.2 \mathrm{mmol}$ sodium phenyltrihydroxyborate, $5 \mathrm{~mL}$ water, $100{ }^{\circ} \mathrm{C}, 0.02 \mathrm{~g}$ Pd/PP-3 $(2 \mathrm{wt} \%$ $\mathrm{Pd}){ }^{\mathrm{b}}$ Yield refer to the isolated product; ${ }^{\mathrm{c}}$ Reaction rate (mol product per mol of total Pd per time) at $10 \mathrm{~min}$;

${ }^{\mathrm{d}}$ Reaction was carried out with $\mathrm{Pd} / \mathrm{MCM}-41 ;{ }^{\mathrm{e}} \mathrm{Pd} / \mathrm{C}$ catalyzed reaction. 
It is worthy to mention that the Suzuki coupling reaction of aromatic chloride by Pd/PP-3 showed almost a $40 \%$ yield of biphenyl in water, which indicates that Pd/PP-3 also has a potential for utilizing much cheaper chloroaromatics for making biphenyl in a cost-effective way. All products of the Suzuki reaction, given in Table 2, were characterized by ${ }^{1} \mathrm{H}$ NMR and ${ }^{13} \mathrm{C}$ NMR (see Supplementary Materials). It is pertinent to mention that the model Suzuki reaction, when compared with Pd/MCM-41 ( $2 \mathrm{wt} \% \cdot \mathrm{Pd} ; 5-8 \mathrm{~nm})$ and $\mathrm{Pd} / \mathrm{C}(2 \mathrm{wt} \% \mathrm{Pd} ; 10-15 \mathrm{~nm})$ in water, shows much lower activity than $\mathrm{Pd} / \mathrm{PP}-3$, signifying the importance of amine functionality decorated in hydrophobic PP-3 for the stabilization of active sites, as well as organometallic intermediates. Similarly, Pd nanoparticles stabilized by phenol resign also show comparable catalytic activity as that of Pd/PP-3, which again proves that the surface functional sites could endow a significant binding interaction with guest metal particles for stabilization and improvement in its catalytic property $[37,38]$.

In Figure $5 c, d$, we present the kinetic aspect of the Pd/PP-3 catalyzed Suzuki reaction, which shows that the reaction is devoid of any long induction time. The reaction rate shows first order dependency with respect to bromobenzene; the rate constant is calculated to be $k_{\mathrm{obs}}=0.081 \pm 0.006 \mathrm{~h}^{-1}$, and the half life period, $t_{1 / 2}$, is $6.93 \mathrm{~h}$. The first order reaction rate can be explained on the basis of an initial oxidative addition by bromobenzene with surface-exposed Pd nanoparticles in a slow step, accompanied by rapid elimination of the cross coupled product.

Finally, the heterogeneity of Pd/PP-3 was proved through a hot filtration test. In this regard, we initially separate the catalyst after the $10 \mathrm{~h}$ reaction is over. The hot filtrate without any Pd/PP-3 was then investigated for the Suzuki reaction, which did not produce any cross coupling products, signifying that the filtrate is devoid of any Pd-based impurity (Figure 6a). Again, ICP measurements of the filtrate solution did not detect any $\mathrm{Pd}$ content, i.e., the $\mathrm{Pd}$ amount in the filtrate is beyond the scope of its detection limit. All these results clearly suggest that Pd sites in Pd/PP-3 are stable for liquid-phase catalytic reactions.
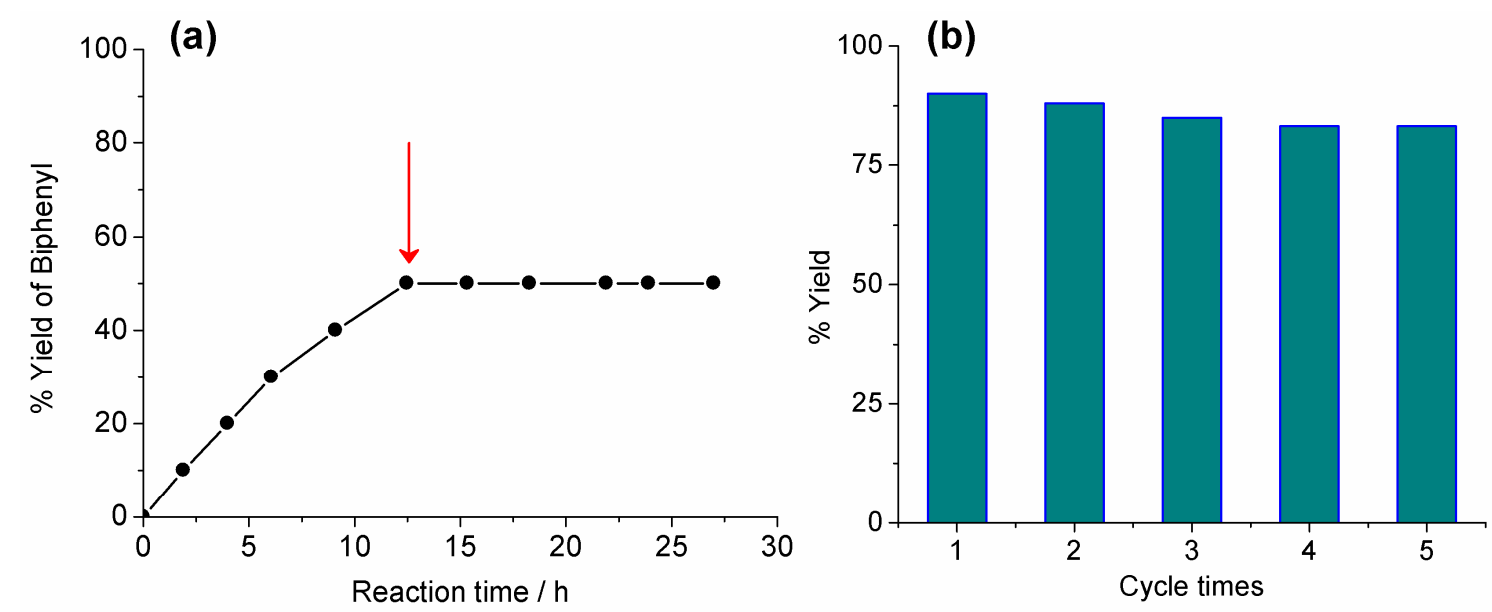

Figure 6. (a) Percent yield of biphenyl versus time during a leaching experiment, where the red arrow indicates the time when catalyst was separated and the supernatant was run afterward; (b) Recycling study of Pd/PP-3 for Suzuki coupling reaction between iodobenzene and sodium salt of phenyltrihydroxyborate.

Furthermore, we studied poisoning experiments in order to check if the reaction is still catalyzed by leached Pd nanoparticles or not. In this regard, we added 2-3 drops of metallic $\mathrm{Hg}$ at the middle of the reaction, and the addition of $\mathrm{Hg}$ hardly affects the rate as well as the overall yield of final product, demonstrating that $\mathrm{Pd} / \mathrm{PP}-3$ has good heterogeneous characteristics for successive reactions.

Later, while investigating the recycling experiments, we found that Pd/PP-3 retains its catalytic activity for five cycles without much deterioration in its activity (Figure 6b). All these results, however, demonstrate that Pd/PP-3 is a stable heterogeneous catalyst for long-term applications. Since Pd/PP-3 
was used to repeat Suzuki coupling experiments in a water medium, there might be another possibility of damage in either morphology or active Pd sites. In this regard, we investigated the stability of reused Pd/PP-3 through TEM and XPS analysis. XPS investigation of the 5th reused Pd/PP-3 shows marginal change in $\mathrm{Pd} 3 \mathrm{~d}$ electrons, possibly because of the partial oxidation or continuous exposure in water (Figure 7). On the other hand, TEM analysis of the 3rd reused Pd/PP-3 suggests no collapse of hollow tube morphology and almost no change in the Pd size distribution (3-6 nm particles), as shown in Figure 8.

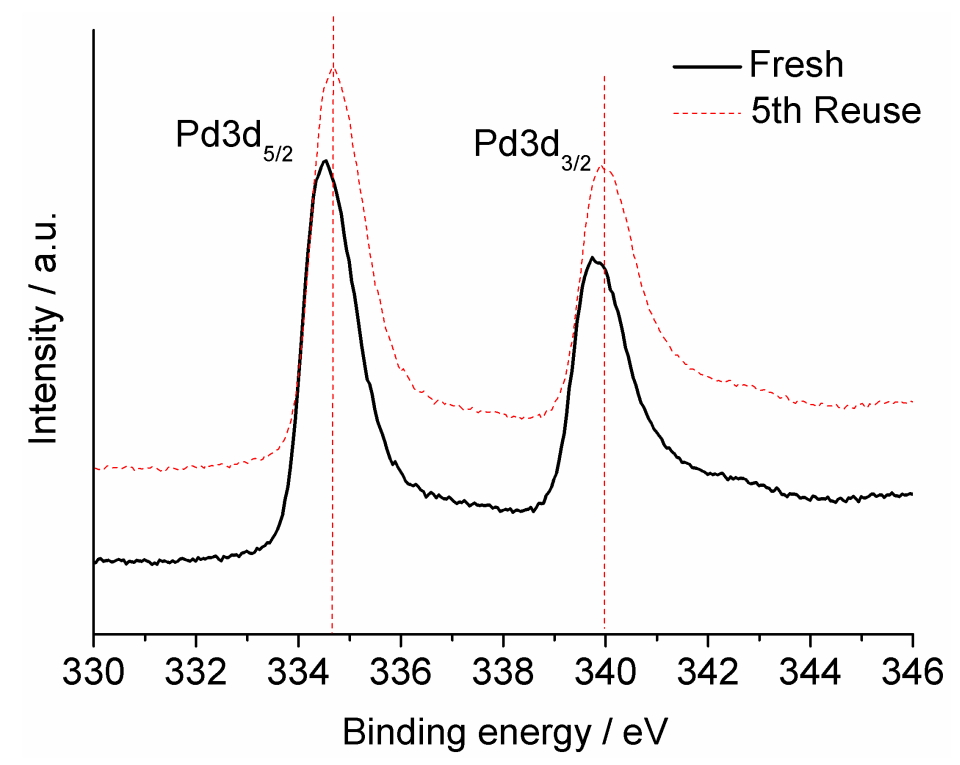

Figure 7. XPS spectra of 5th recycled Pd/PP-3 catalyst.

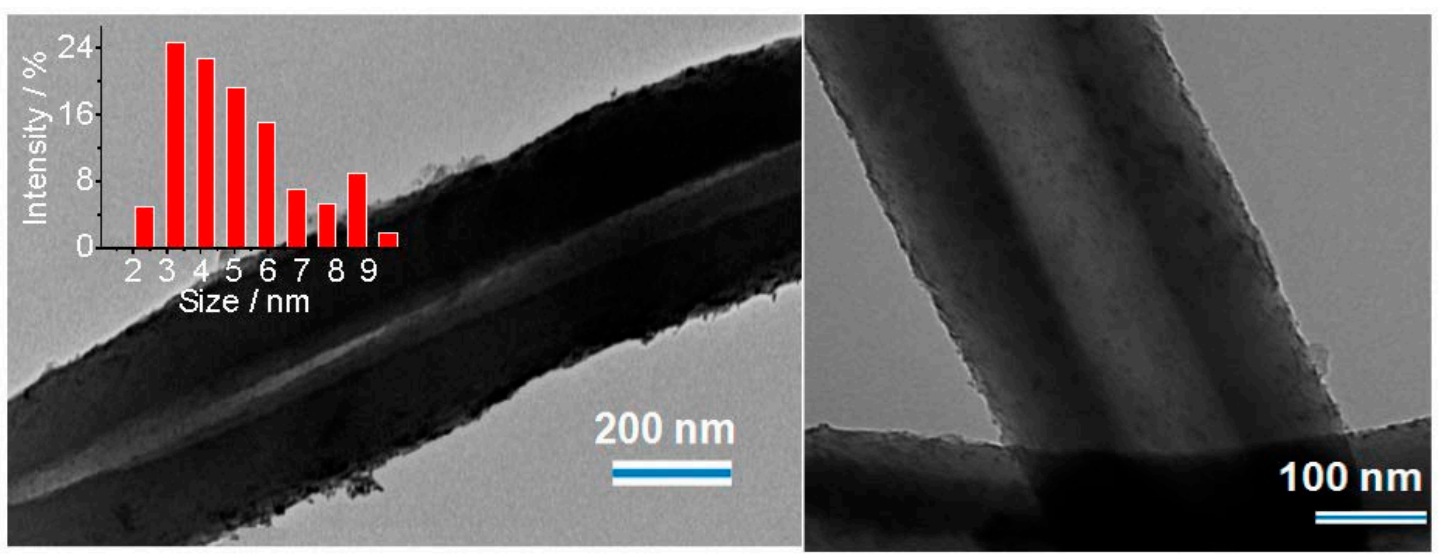

Figure 8. TEM of Pd/PP-3 after third catalytic cycle is over.

\section{Materials and Methods}

\subsection{Instrumentation}

Porosity was measured at $77 \mathrm{~K}$ using a Quantachrome Instrument (Quantachrome Instrument; Boynton Beach, FL, USA), Autosorb-1, where all samples was degassed at $100{ }^{\circ} \mathrm{C}$ for $4 \mathrm{~h}$ before the measurement. The Brauner-Emmett-Teller (BET) surface area was calculated over the entire pressure region from $\sim 0.05$ to $\sim 0.18 \mathrm{P} / \mathrm{P}_{0}$. Transmission electron microscopy (TEM) was obtained from Hitachi HT-7700 (Nishi-shimbashi, Minato-Ku, Tokyo, Japan) with an acceleration voltage at $100 \mathrm{kV}$ after the samples were dispersed in ethanol via sonication and placed onto an ultrathin carbon film supported 
on a copper grid. Powder X-ray diffraction (PXRD) was performed with a Rigaku D/Max2500 PC diffractometer with $\mathrm{CuK} \alpha$ radiation $(\lambda=1.5418 \AA)$ over the $2 \theta$ range of $5^{\circ}-70^{\circ}$ at a scan speed of $5^{\circ}$ per min at room temperature. A Bruker DPX-300 NMR spectrometer was used to measure the ${ }^{1} \mathrm{H}$ and ${ }^{13} \mathrm{C}$ NMR of catalytic products in a liquid state. X-ray photoelectron spectroscopy (XPS) was recorded on a VG ESCALAB MK2 apparatus using AlK $\alpha(h v=1486.6 \mathrm{eV})$ as the excitation light source. Pd content was determined via PLASAM-SPEC-II inductively coupled plasma atomic emission spectrometry (ICP). Particle sizes were determined from Nano Measurer 1.2 software, 2008 by Jie Xu, Fudan University, China.

\subsection{Methods}

Synthesis of PP-3 was followed in accordance with a previously reported procedure [24]. For the preparation of Pd/PP-3, we initially dissolved $0.010 \mathrm{~g}$ of palladium acetate in a $20 \mathrm{~mL}$ glass vial containing $10 \mathrm{~mL}$ of distilled water and stirred until the solution became yellow. Next, $0.05 \mathrm{~g}$ of PP-3 was mixed, and the solution was stirred for $7 \mathrm{~h}$. The mixture was centrifuged several times and washed with $\mathrm{H}_{2} \mathrm{O}$ and ethanol followed by drying at $60{ }^{\circ} \mathrm{C}$ for $12 \mathrm{~h}$, which was denoted as $\mathrm{Pd} / \mathrm{PP}-3$. Pd loading was found to be $\sim 2 \mathrm{wt} \%$, as confirmed by ICP analysis.

\subsection{General Procedure for Suzuki-Miyaura Coupling Reaction}

In the typical synthesis condition, a mixture of Arylhalide $(1 \mathrm{mmol})$, sodium phenyltrihydroxyborate (1.2 mmol), $0.02 \mathrm{~g} \mathrm{Pd} /$ PP-3 (2 wt \% Pd), and TBAB (1 mmol) was stirred in water $(5 \mathrm{~mL})$. The mixture was refluxed with magnetic stirring for several hours, as shown in Table 2. After the reaction was complete (monitored by thin layer chromatography technique), the mixture was filtered to separate the catalyst, and the filtrate was subjected to extract (10-20 min) with diethyl ether $(20 \mathrm{~mL})$. The combined organic layers were then washed with brine $(10 \mathrm{~mL})$, dried by anhydrous $\mathrm{Na}_{2} \mathrm{SO}_{4}$, and evaporated. The residue was purified on a short column of silica using petroleum ether as the eluent to afford the desired substituted biphenyl as pure product.

\section{Conclusions}

Herein, we report on a Suzuki-Miyaura cross coupling reaction in water with Pd-grafted PP-3 as a porous polymer support, which is thought to be promising and environmentally appealing. High catalytic activity, good stability, and reusability of Pd/PP-3 essentially signify its advantages as a heterogeneous catalyst for the liquid phase synthesis of fine chemicals. Moreover, the hollow tube geometry of PP-3 is suitable for anchoring Pd sites by exploiting both the inner and outer hollow spaces, providing an enormous stabilization of $\mathrm{Pd}$ and preventing the formation of inactive Pd clusters. Owing to the importance of particle morphologies in solid catalytic research, we believe our efforts could motivate others' for developing organic nanotubes/carbon tubes and utilization of its hollow space for developing nanoreactors, which could ultimately lead to a sustainable and environmentally benign solid catalysis research.

Supplementary Materials: The following are available online at www.mdpi.com/2073-4344/6/10/161/s1.

Acknowledgments: We acknowledge the National Natural Science Foundation of China (No. 21276191), the Specialized Research Fund for the Doctoral Program of Higher Education of China (No. 20120032120083), the Natural Science Foundation of Tianjin, China (No. 16JCQNJC06200) for financial support.

Author Contributions: A.M. designed scheme and experiments; A.M., J.S. \& W.Q. performed all experiments and collected data; X.L. \& A.M. analyzed the data and finally wrote the manuscript.

Conflicts of Interest: The authors declare no conflict of interest.

\section{References}

1. Kaur, P.; Hupp, J.T.; Nguyen, S.T. Porous Organic Polymers in Catalysis: Opportunities and Challenges. ACS Catal. 2011, 1, 819-835. [CrossRef] 
2. Modak, A.; Pramanik, M.; Inagaki, S.; Bhaumik, A. A triazine functionalized porous organic polymer: excellent $\mathrm{CO}_{2}$ storage material and support for designing $\mathrm{Pd}$ nanocatalyst for $\mathrm{C}-\mathrm{C}$ cross-coupling reactions. J. Mater. Chem. A 2014, 2, 11642-11650. [CrossRef]

3. Sun, Q.; Dai, Z.; Meng, X.; Wang, L.; Xiao, F.S. Task-Specific Design of Porous Polymer Heterogeneous Catalysts beyond Homogeneous Counterparts. ACS Catal. 2015, 5, 4556-4567. [CrossRef]

4. Zhang, P.; Weng, Z.; Guo, J.; Wang, C. Solution-Dispersible, Colloidal, Conjugated Porous Polymer Networks with Entrapped Palladium Nanocrystals for Heterogeneous Catalysis of the Suzuki-Miyaura Coupling Reaction. Chem. Mater. 2011, 23, 5243-5249. [CrossRef]

5. Modak, A.; Mondal, J.; Bhaumik, A. Highly Porous Organic Polymer containing Free $-\mathrm{CO}_{2} \mathrm{H}$ Groups: A Convenient Carbocatalyst for Indole C-H Activation at Room Temperature. ChemCatChem 2013, 5, 1749-1753. [CrossRef]

6. Zhang, Y.; Riduan, S.N. Functional porous organic polymers for heterogeneous catalysis. Chem. Soc. Rev. 2012, 41, 2083-2094. [CrossRef] [PubMed]

7. Sprick, R.S.; Jiang, J.X.; Bonillo, B.; Ren, S.; Ratvijitvech, T.; Guiglion, P.; Zwijnenburg, M.A.; Adams, D.J.; Cooper, A.I. Tunable Organic Photocatalysts for Visible-Light-Driven Hydrogen Evolution. J. Am. Chem. Soc. 2015, 137, 3265. [CrossRef] [PubMed]

8. Modak, A.; Yamanaka, K.I.; Goto, Y.; Inagaki, S. Photocatalytic $\mathrm{H}_{2}$ Evolution by Pt-Loaded 9,9'-Spirobifluorene-Based Conjugated Microporous Polymers under Visible-Light Irradiation. Bull. Chem. Soc. Jpn. 2016, 89, 887-891. [CrossRef]

9. Liras, M.; Marta Iglesias, M.; Félix Sánchez, F. Conjugated Microporous Polymers Incorporating BODIPY Moieties as Light-Emitting Materials and Recyclable Visible-Light Photocatalysts. Macromolecules 2016, 49, 1666-1673. [CrossRef]

10. Zhou, H.C.J.; Kitagawa, S. Metal-Organic Frameworks (MOFs). Chem. Soc. Rev. 2014, 43, 5415-5418. [CrossRef] [PubMed]

11. Liu, X.; Maegawa, Y.; Goto, Y.; Hara, K.; Inagaki, S. Heterogeneous Catalysis for Water Oxidation by an Iridium Complex Immobilized on Bipyridine-Periodic Mesoporous Organosilica. Angew. Chem. Int. Ed. 2016, 55, 7943-7947. [CrossRef] [PubMed]

12. Modak, A.; Mondal, J.; Aswal, V.K.; Bhaumik, A. A new periodic mesoporous organosilica containing diimine-phloroglucinol, $\mathrm{Pd}(\mathrm{II})$-grafting and its excellent catalytic activity and trans-selectivity in C-C coupling reactions. J. Mater. Chem. 2010, 20, 8099-8106. [CrossRef]

13. Jagtap, S.; Deshpande, R. True water soluble palladium-catalyzed Heck reactions in aqueous-organic biphasic media. Tetrahedron Lett. 2013, 54, 2733-2736. [CrossRef]

14. Gilbert, L.; Mercier, C. Solvent effects in heterogeneous catalysis: Application to the synthesis of fine chemicals. Stud. Surface Sci. Catal. 1993, 78, 51-66.

15. Shaughnessy, K.H.; DeVasher, R.B. Palladium-Catalyzed Cross-Coupling in Aqueous Media: Recent Progress and Current Applications. Curr. Org. Chem. 2005, 9, 595. [CrossRef]

16. Selander, N.; Szabó, K.J. Catalysis by Palladium Pincer Complexes. Chem. Rev. 2011, 111, $2048-2076$. [CrossRef] [PubMed]

17. Miyaura, N.M.; Suzuki, A. Palladium-Catalyzed Cross-Coupling Reactions of Organoboron Compounds. Chem. Rev. 1995, 95, 2457-2483. [CrossRef]

18. Masjedi, M.; Demiralp, T.; Özkar, S. Testing catalytic activity of ruthenium(III) acetylacetonate in the presence of trialkylphosphite or trialkylphosphine in hydrogen generation from the hydrolysis of sodium borohydride. J. Mol. Catal. A Chem. 2009, 310, 59-63. [CrossRef]

19. Chen, L.; Gao, Z.; Li, Y. Immobilization of Pd(II) on MOFs as a highly active heterogeneous catalyst for Suzuki-Miyaura and Ullmann-type coupling reactions. Catal. Today 2015, 245, 122-128. [CrossRef]

20. Li, B.; Guan, Z.; Wang, W.; Yang, X.; Hu, J.; Tan, B.; Li, T. Highly Dispersed Pd Catalyst Locked in Knitting Aryl Network Polymers for Suzuki-Miyaura Coupling Reactions of Aryl Chlorides in Aqueous Media. Adv. Mater. 2012, 24, 3390-3395. [CrossRef] [PubMed]

21. Kumbhar, A.; Kamble, S.; Mane, A.; Jha, R.; Salunkhe, R. Modified zeolite immobilized palladium for ligand-free Suzuki-Miyaura cross-coupling reaction. J. Organomet. Chem. 2013, 738, 29-34. [CrossRef]

22. Gokmen, M.T.; Prez, F.E.D. Porous polymer particles-A comprehensive guide to synthesis, characterization, functionalization and applications. Prog. Polym. Sci. 2012, 37, 365-405. [CrossRef] 
23. Feng, X.; Liang, Y.; Zhi, L.; Thomas, A.; Wu, D.; Lieberwirth, I.; Kolb, U.; Mullen, K. Synthesis of Microporous Carbon Nanofibers and Nanotubes from Conjugated Polymer Network and Evaluation in Electrochemical Capacitor. Adv. Funct. Mater. 2009, 19, 2125-2129. [CrossRef]

24. Modak, A.; Bhaumik, A. High-throughput Acid-Base Tandem Organocatalysis over Hollow Tube-Shaped Porous Polymers and Carbons. ChemistrySelect 2016, 6, 1192-1200. [CrossRef]

25. Kang, N.; Hon Park, J.; Choi, J.; Jin, J.; Chun, J.; Jung, I.G.; Jeong, J.; Geun Park, J.; Lee, S.M.; Kim, H.J.; et al. Nanoparticulate Iron Oxide Tubes from Microporous Organic Nanotubes as Stable Anode Materials for Lithium Ion Batteries. Angew. Chem. Int. Ed. 2012, 51, 6626-6630. [CrossRef] [PubMed]

26. Mukhopadhyay, K.; Phadtare, S.; Vinod, V.P.; Kumar, A.; Rao, M.; Chaudhari, R.V.; Sastry, M. Gold Nanoparticles Assembled on Amine-Functionalized Na-Y Zeolite: A Biocompatible Surface for Enzyme immobilizaiton. Langmuir 2003, 19, 3858-3863. [CrossRef]

27. Mandal, S.; Roy, D.; Chaudhari, R.V.; Sastry, M. Pt and Pd Nanoparticles Immobilized on Amine-Functionalized Zeolite: Excellent Catalysts for Hydrogenation and Heck Reactions. Chem. Mater. 2004, 16, 3714-3724. [CrossRef]

28. Huang, Y.; Zheng, Z.; Liu, T.; Lü, J.; Lin, Z.; Li, H.; Cao, R. Palladium nanoparticles supported on amino functionalized metal-organic frameworks as highly active catalysts for the Suzuki-Miyaura cross coupling reactions. Catal. Commun. 2011, 14, 27-31. [CrossRef]

29. Dai, B.; Wen, B.; Zhu, M.; Kanga, L.; Yu, F. Nickel catalysts supported on amino functionalized MCM-41 for syngas methanation. RSC Adv. 2016, 6, 66957-66962. [CrossRef]

30. Zhu, Q.L.; Tsumori, N.; Xu, Q. Immobilizing Extremely Catalytically Active Palladium Nanoparticles to Carbon Nanospheres: A Weakly-Capping Growth Approach. J. Am. Chem. Soc. 2015, 137, 11743-11748. [CrossRef] [PubMed]

31. Huang, N.; Xu, Y.; Jiang, D. High-performance heterogeneous catalysis with surface-exposed stable metal nanoparticles. Sci. Rep. 2014, 4, 7228. [CrossRef] [PubMed]

32. Ohtaka, A.; Okagaki, T.; Hamasaka, G.; Uozumi, Y.; Shinagawa, T.; Shimomura, O.; Nomura, R. Application of "Boomerang" Linear Polystyrene-Stabilized Pd Nanoparticles to a Series of C-C Coupling Reactions in Water. Catalysts 2015, 5, 106-118. [CrossRef]

33. Wang, C.; Yang, F.; Yang, W.; Ren, L.; Zhang, Y.; Jia, X.; Zhang, L.; Li, Y.F. PdO nanoparticles enhancing the catalytic activity of $\mathrm{Pd}$ /carbon nanotubes for 4-nitrophenol reduction. RSC Adv. 2015, 5, 27526-27532. [CrossRef]

34. Cammidge, A.N.; Goddard, V.H.M.; Gopee, H.; Harrison, N.L.; Hughes, D.L.; Schubert, C.J.; Sutton, B.M.; Watts, G.L.; Whitehead, A.J. Aryl Trihydroxyborates: Easily Isolated Discrete Species Convenient for Direct Application in Coupling Reactions. Org. Lett. 2006, 8, 4071. [CrossRef] [PubMed]

35. Phan, T.S.; Van Der Sluys, M.; Jones, C.W. On the Nature of the Active Species in Palladium Catalyzed Mizoroki-Heck and Suzuki-Miyaura Couplings-Homogeneous or Heterogeneous Catalysis, A Critical Review. Adv. Synth. Catal. 2006, 348, 609. [CrossRef]

36. Rostamnia, S.; Alamgholiloo, H.; Liu, X. Pd-grafted open metal site copper-benzene-1,4-dicarboxylate metal organic frameworks (Cu-BDC MOF's) as promising interfacial catalysts for sustainable Suzuki coupling. J. Colloid Interface Sci. 2016, 469, 310-317. [CrossRef] [PubMed]

37. Xu, T.Y.; Zhang, Q.F.; Yang, H.F.; Li, X.N.; Wang, J.G. Role of Phenolic Groups in the Stabilization of Palladium Nanoparticles. Ind. Eng. Chem. Res. 2013, 52, 9783-9789. [CrossRef]

38. Nishiwaki, N.; Hamada, S.; Watanabe, T.; Hirao, S.; Jun Sawayama, J.; Asahara, H.; Saigo, K.; Kamata, T.; Funabashi, M. Development of a new palladium catalyst supported on phenolic resin. RSC Adv. 2015, 5, 4463-4467. [CrossRef]

(C) 2016 by the authors; licensee MDPI, Basel, Switzerland. This article is an open access article distributed under the terms and conditions of the Creative Commons Attribution (CC-BY) license (http:/ / creativecommons.org/licenses/by/4.0/). 